\title{
Progress in semiconductor quantum dots-based continuous-wave laser
}

\author{
Lei Wang $^{1^{*}}$, Guang Dai ${ }^{2}$, Luogen Deng ${ }^{2}$ and Haizheng Zhong ${ }^{1^{*}}$
}

\begin{abstract}
Continuous-wave (CW) operated laser is a key component for photonic chips. It has been highly desired to develop low-threshold, high-efficiency, long-term operational, low-cost and easily integratable CW laser. As excellent optical gain materials, quantum dots (QDs) have been intensively investigated for on-chip $\mathrm{CW}$ lasers owing to their high photoluminescence quantum yields, tunable emission wavelengths and easy integration. Base on the difference of preparation processes, QDs can be classified into epitaxial QDs and solution-processed colloidal QDs (CQDs). In this mini-review, we summarize the research progresses of epitaxial III-V semiconductor QD and solution-processed CQD-based CW lasers. The challenges associated with the realization of CQD CW lasers are discussed in detail. In particular, the emerging perovskite-based CW lasers are highlighted. Finally, a short perspective of QD-based CW lasers is presented.
\end{abstract}

Keywords: quantum dots, continuous-wave laser, perovskite, photonics

\section{INTRODUCTION}

In response to the explosive growth of global information, the high-speed, high-density and low-cost information processing technique is intensively needed [1]. Photonic chips hold great promise to be the next generation data processing technology due to their fast responsibility. As described by Paul [2], a photonic chip at least includes the components with functionalities of light generation, transportation, and detections. Over the past decades, great success has been made in the on-chip light sources, high-bandwidth detectors, fast modulators and waveguides [3-8]. In comparison, the development of on-chip light source lags behind the other counterparts, which has become a great challenge for the field of integrated photonics.

Continuous-wave (CW) lasers have been considered a remarkable breakthrough to achieve on-chip integrated light source. The epitaxial growth of III-V semiconductor quantum wells (QWs) on silicon ( $\mathrm{Si}$ ) substrates has enabled CW laser for chip-to-chip optical communications. However, the performance is limited due to the lattice mismatch and the thermal expansion coefficient difference between III-V materials and $\mathrm{Si}$ substrates [3]. The III-V quantum dots (QDs) have ability to overcome these hindrances [9-14]. The performance of III-V QD CW laser has been greatly improved in the past few years, which approaches the requirements of on-chip light source in near infrared (NIR) region. Along with that, the demand of CW laser also promotes the research of colloidal QDs (CQDs) as economical and color-tunable alternatives for CW laser applications [15]. The gain thresholds of CQD-based CW lasers have been greatly reduced by means of materials engineering. However, the thresholds are still far from the practical application. Recently, the halide perovskites are emerging as alternative gain media to reduce the threshold to achieve CW lasers [16-23]. The primary results have encouraged the entire community to pursue perovskite-based roomtemperature CW lasing.

In this mini-review, we summarize the recent developments of CW lasers based on epitaxial QDs and solution-processed CQDs. In the first part, we describe the progress of III-V semiconductor QD-based CW lasers on different substrates, including germanium $(\mathrm{Ge}), \mathrm{Ge}$-on-Si and $\mathrm{Si}$ substrates with a following discussion about micro-disk (MD) CW lasers. In the second part, we review

${ }^{1}$ MIIT Key Laboratory for Low-dimensional Quantum Structure and Devices, School of Materials Science \& Engineering, Beijing Institute of Technology, Beijing 100081, China

2 School of Physics, Beijing Institute of Technology, Beijing 100081, China

* Corresponding authors (emails: hzzhong@bit.edu.cn (Zhong H); wanglei195512@126.com (Wang L)) 
the advancement in the field of solution-processed CQDbased CW lasers and discuss the current challenges for room-temperature-operated CQD CW lasers. In the third part, the recent perovskite-based CW lasers are presented and discussed. Finally, we give a short conclusion with our perspectives for the future investigation. Instead of a comprehensive description, we try to give the milestones and future trends of QD-based CW lasers.

\section{III-V SEMICONDUCTOR QD CW LASERS}

The room-temperature-operated semiconductor CW lasing was firstly achieved using AlAs-GaAs heterostructure QW by Alferov et al. [24] in 1971. Since then, CW lasers based on QWs have been extensively explored. However, their thresholds are temperature sensitive. To solve this problem, Arakawa et al. [25] theoretically proposed the use of QDs as gain media to decrease the temperature sensitive threshold in 1982. Twelve years later, Kirstaedter et al. [26] fabricated the first QD-based CW laser using the Stranski-Krastanow growth method. They observed room-temperature lasing from ( $\mathrm{InGa}) \mathrm{As}$ QDs with a threshold current density of $950 \mathrm{~A} \mathrm{~cm}^{-2}$. From then on, the development of QD-based CW lasers has drawn a great number of attentions from both of scientific and industrial fields.

The zero-dimensional (0D) QDs can more easily achieve population inversion compared with $2 \mathrm{D}$ or $3 \mathrm{D}$ materials. Semiconductor QDs represent particle-in-abox-like quantum-confined structures, exhibiting atomor molecule-like properties [27]. At quantum length scale, the electronic spectra of QDs show discrete density of states due to the quantum confinement effect, tending to delta-function-like energy levels. Similar to atoms, the ground state of QDs is doubly degenerated, which has a maximum occupancy of two electrons. Therefore, the $0 \mathrm{D}$ QDs have the lowest achievable gain threshold. Because of the high energy level spacing between the discrete energy levels, the carrier population is restrained to broaden into higher states [27]. Besides, the thermal spreading of carriers in QDs is decreased due to the deltafunction like state density. These characters make the threshold of QD lasers be far less temperature sensitive than that of conventional QW lasers [25].

With the efforts from all over the world, the performance of III-V QD-based CW laser has been greatly improved. Table 1 summarizes the milestones in the developments. In the following, we will describe the details in the material innovations on the growth.

\section{III-V semiconductor QD CW lasers on Ge or Ge-on-Si substrates}

In 2000, Kazi et al. [28] fabricated the self-formed InGaAs

Table 1 Representative milestones of epitaxial III-V semiconductor QD-based CW lasers

\begin{tabular}{|c|c|c|c|c|c|c|c|}
\hline Authors & Description & $\begin{array}{l}\text { Wavelength } \\
\text { (nm) }\end{array}$ & $\begin{array}{c}\text { Thresholds } \\
\left(\mathrm{A} \mathrm{cm}^{-2}\right)\end{array}$ & $\begin{array}{l}\text { Operation } \\
\text { condition }\end{array}$ & $T_{\max }^{\mathrm{b}}\left({ }^{\circ} \mathrm{C}\right)$ & Lifetime (h) & Publication year \\
\hline Arakawa et al. [25] & Theoretically proposed & & & & & & 1982 \\
\hline Kazi [28] & $\mathrm{In}_{0.2} \mathrm{Ga}_{0.8}$ As 'QD like' & 854 & 1320 & $\mathrm{RT}^{\mathrm{a}}$ & & 78 & 2000 \\
\hline \multicolumn{8}{|c|}{ On Ge or Ge-on-Si substrates } \\
\hline Liu et al. [29] & InAs/GaAs QDs On Ge & 1305 & 55.2 & RT & 60 & & 2011 \\
\hline Lee et al. [30] & InAs/GaAs QDs On Ge/Si & 1280 & 163 & RT & 30 & & 2012 \\
\hline Liu et al. [31] & InAs on $\mathrm{Ge} / \mathrm{Si}$ & $\sim 1250$ & $\sim 426$ & $\mathrm{RT}$ & 119 & & 2014 \\
\hline Liu et al. [32] & InAs/GaAs on $\mathrm{Ge} / \mathrm{Si}$ & 1267 & 2000 & RT & 30 & $\begin{array}{c}4600 \\
\text { (extrapolated) }\end{array}$ & 2015 \\
\hline \multicolumn{8}{|l|}{ On Si substrates } \\
\hline Chen et al. [3] & InAs/GaAs on $\mathrm{Si}$ & 1315 & 62.5 & $\mathrm{RT}$ & 75 & $\begin{array}{c}10,0158 \\
\text { (extrapolated) }\end{array}$ & 2016 \\
\hline Liu et al. [9] & InAs/GaAs on $\mathrm{GaP} / \mathrm{Si}$ & 1280 & 860 & RT & 90 & & 2017 \\
\hline Norman et al. [12] & InAs on $\mathrm{V}$-groove $\mathrm{Si}$ & 1250 & 333 & RT & 80 & & 2017 \\
\hline Chen et al. [13] & InAs/GaAs on $\mathrm{Si}$ & 1292 & 425 & RT & 36 & & 2017 \\
\hline Jung et al. $[10]$ & InAs on $\mathrm{GaP} / \mathrm{Si}$ & 1270 & 132 & RT & 80 & & 2017 \\
\hline Wang et al. [33] & InAs/GaAs on $\mathrm{Si}$ & $\sim 1300$ & 550 & RT & & & 2018 \\
\hline
\end{tabular}

a, b) RT and $T_{\max }$ represent room-temperature and the maximum lasing temperature, respectively. 
QD-like structures on Si substrate by the metalorganic chemical vapor deposition (MOCVD) method. The atomic force microscopy (AFM) characterizations show that the as-formed InGaAs QD-like nanocrystals have a diameter of about $20 \mathrm{~nm}$ with an average height of $\sim 6 \mathrm{~nm}$. The laser structure was composed of a QD-like active region between 70-nm-thick $\mathrm{Al}_{0: 3} \mathrm{Ga}_{0: 7}$ As layers. An electrically pumped CW laser at wavelength of $\sim 860 \mathrm{~nm}$ with a threshold of $1.32 \mathrm{kA} \mathrm{cm}^{-2}$ was achieved. CW laser at optical communication wavelength has been of great interest. The breakthrough of III-V QDs at about $1300 \mathrm{~nm}$ was accomplished by Liu et al. [29] in 2011. They proposed the growth of single-domain GaAs buffer layers on the Ge substrate using Ga pre-layer technique. The AFM and transmission electron microscopy (TEM) images show that the obtained GaAs layer has a quite smooth surface morphology (Fig. 1a). With that, InAs/ GaAs QDs can be fabricated directly on the pre-fabricated high-quality GaAs buffer layer. By using this method, the antiphase boundary (APB) issues were resolved, and the room-temperature-operated $\mathrm{CW}$ lasing at wavelength of $1305 \mathrm{~nm}$ with a low threshold of $55.2 \mathrm{~A} \mathrm{~cm}^{-2}$ was observed (Fig. $1 b$ and c).

Soon after the demonstration of InAs/GaAs QD CW laser on Ge substrate, the room-temperature InAs/GaAs QD CW laser on Ge-on-Si substrates was reported by the same group. The threshold was about $163 \mathrm{~A} \mathrm{~cm}^{-2}$ [30]. Using a similar method, Liu et al. [31] fabricated p-doped InAs QDs epitaxially grown on Ge-on-Si substrate in 2014. High temperature CW lasing emission with a threshold of $\sim 255 \mathrm{~mA}$ was obtained at a temperature up to $119^{\circ} \mathrm{C}$. Furthermore, they studied the InAs/GaAs selfassembled QD lasers epitaxially grown on the Ge-on-Si substrates. The obtained $\mathrm{CW}$ laser was able to operate over $2700 \mathrm{~h}$ at $30^{\circ} \mathrm{C}[32]$.

\section{III-V semiconductor QD CW lasers on Si substrates}

Although the QD CW lasers on Ge or Ge-on-Si substrates show excellent performances, the QD CW lasers directly grown on Si substrates without the intermediate Ge layer are more attractive because the Ge layer restricts the range of $\mathrm{Si}$ circuits and has large optical absorption coefficient at telecommunication wavelengths $[3,33]$. However, it is difficult to grow CW lasers directly on $\mathrm{Si}$ due to the lattice mismatch and the thermal expansion coefficient difference between III-V materials and $\mathrm{Si}$ substrates [3]. The threading dislocation (TD) in the gain layers forms a number of non-radiative recombination centers and cuts down the laser performance. QDs are less sensitive to defects. A breakthrough of QD CW lasers directly grown on Si was firstly accomplished by Chen et al. [3] in 2016. In their work, they developed several important strategies to realize highly efficient monolithic QD lasers. Initially, they used the phosphorus-doped $\mathrm{Si}$ (100) wafers with $4^{\circ}$ offcut to the [011] plane to prevent the formation of antiphase domains when growing III-V materials on Si substrates. Then, they applied a thin AlAs layer to provide a good interface for III-V QDs growth. Furthermore, the $\mathrm{In}_{0.18} \mathrm{Ga}_{0.82} \mathrm{As} / \mathrm{GaAs}$ strained-layer superlattices were grown as dislocation filter layers on the GaAs buffer layer. Taking advantages of these efforts, they successfully fabricated room-temperature-operated QD CW lasers with a low threshold of $62.5 \mathrm{~A} \mathrm{~cm}^{-2}$, high output power of $>105 \mathrm{~mW}$, and long lifetime operation of $3100 \mathrm{~h}$ (Fig. 2a-c).

The standard Si-based complementary metal-oxidesemiconductor (CMOS) foundry process requires nominal (001) Si substrates. To adapt CMOS processing,
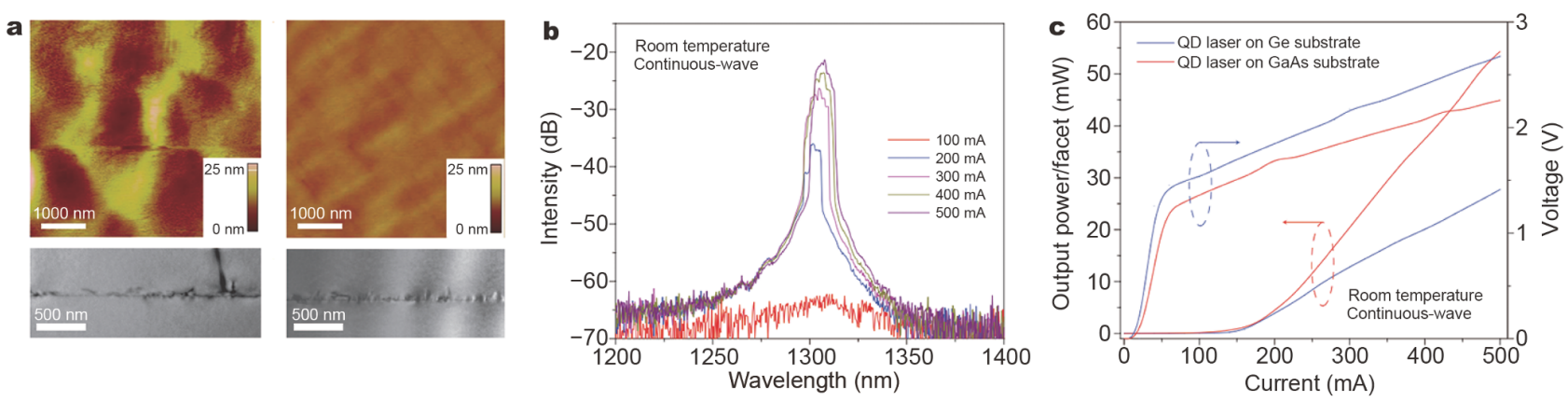

Figure 1 (a) AFM images (top) of the surface morphology for $1.2 \mathrm{~mm} \mathrm{GaAs}$ on Ge substrates with As prelayer (left) and Ga prelayer (right) growth techniques. TEM images (bottom) of the interface between the GaAs buffer layer and the Ge substrate with As prelayer (left) and Ga prelayer (right) growth techniques. (b) Room-temperature emission spectra of the Ge-based InAs/GaAs QD laser for different drive currents below and above threshold. (c) Light output power versus current and voltage versus current for InAs/GaAs QD laser diodes grown on Ge and GaAs substrates. Reproduced with permission from [29]. Copyright 2011, Springer Nature. 

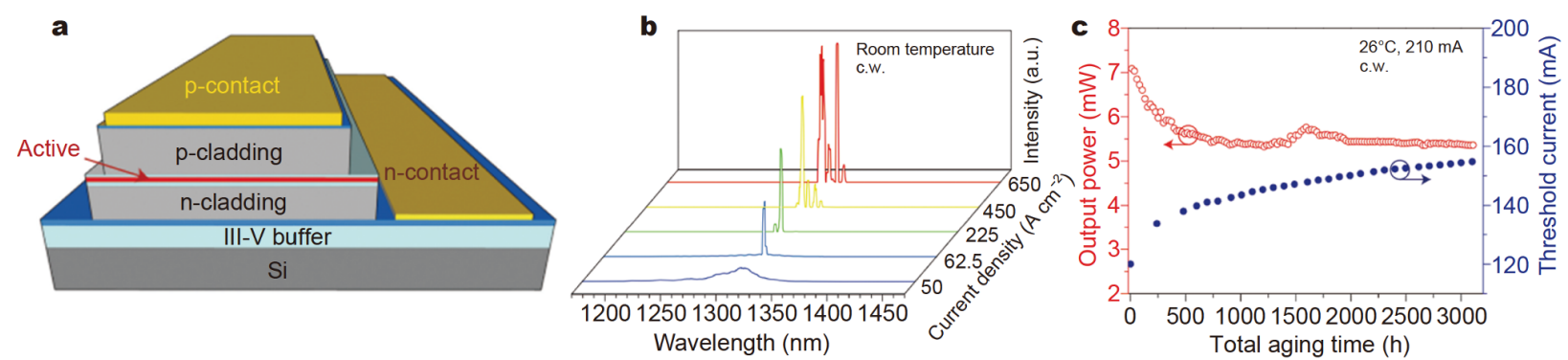

Figure 2 (a) Schematic of the layer structure of an InAs/GaAs QD laser on a Si substrate. (b) Emission spectra for a $50 \mu \mathrm{m} \times 3200 \mu \mathrm{m}$ InAs/GaAs QD laser at various injection current densities under $\mathrm{CW}$ operation at $18^{\circ} \mathrm{C}$. (c) Aging data for the InAs/GaAs QD laser on $\mathrm{Si}$ at a constant heatsink temperature of $26^{\circ} \mathrm{C}$ and CW drive current of $210 \mathrm{~mA}$. Reproduced with permission from [3]. Copyright 2016, Springer Nature.

it is of great industrial importance to grow the III-V semiconductor CW lasers on on-axis (001) Si substrate [9]. In 2017, Liu et al. [9] reported the electrically pumped III-V InAs/GaAs QD CW lasers epitaxially grown on on-axis (001) Si without offcut or Ge layers. The CW laser was developed on an on-axis p-doped $\mathrm{Si}$ substrate with an n-doped Si homo-epitaxial buffer and $\mathrm{n}$-doped $\mathrm{GaP}$ nucleation layer. A threshold around $860 \mathrm{~A} \mathrm{~cm}^{-2}$ and an output power of $110 \mathrm{~mW}$ were achieved. Subsequently, Jung et al. [10] improved the GaAs templates with a TD density of $7.3 \times 10^{6} \mathrm{~cm}^{-2}$ and achieved CW lasing emission with lowest threshold current density of $132 \mathrm{~A} \mathrm{~cm}^{-2}$. In 2018, the group further improved the performance and reliability of QD lasers by introducing a low TD density GaAs buffer layer. The $\mathrm{CW}$ laser with a threshold of $6.2 \mathrm{~mA}$ and an extrapolated mean-time-to-failure of more than one million hours was demonstrated [11].

Another alternative method to realize the $\mathrm{CW}$ lasers directly grown on $\mathrm{Si}$ is using patterned on-axis (001) Si substrate. The patterned on-axis (001) Si could circumvent the issue of antiphase domains and reduce the TD density in the GaAs buffer layer. In 2017, Norman et al. [12] demonstrated the electrically injected CW QD lasers grown on on-axis (001) Si patterned with (111) Vgrooves. They realized the $\mathrm{CW}$ lasing with a threshold of $36 \mathrm{~mA}$ and operation temperature up to $80^{\circ} \mathrm{C}$. The electrically pumped InAs/GaAs QD CW laser monolithically grown on on-axis Si (001) substrates without any buffer layers was also reported [13]. In this work, a $400 \mathrm{~nm}$ APB-free epitaxial GaAs film with a thickness of $400 \mathrm{~nm}$ was first deposited on a standard industrycompatible on-axis Si (001) substrate by MOCVD, and then the laser structure was grown on this APB-free $\mathrm{GaAs} / \mathrm{Si}$ (001) substrate by molecular beam epitaxy [13]. The CW lasing emission with a threshold of $425 \mathrm{~A} \mathrm{~cm}^{-2}$ was achieved.

\section{III-V semiconductor QD MD CW laser on Si substrates} MD can provide whispering-gallery (WG) lasing modes with high quality. In 2016, Wan et al. [34] first demonstrated the optical pumped $1.3 \mu \mathrm{m}$ room-temperature QD MD CW lasers epitaxially grown on pre-patterned onaxis (001) Si substrate without any buffer layer. The MD CW laser consisted a five-layer InAs/InGaAs dot-in-awell structure in a disk region. The tilted and top-down scanning electron microscopy (SEM) images of the disk reveal the smooth sidewall surface of the MD (Fig. 3a). The high quality MD guarantees the achievement of single mode laser with low threshold of $200 \mu \mathrm{W}$, as shown in Fig. 3b and c. In 2017, Kryzhanovskaya et al. [35] demonstrated an electrically pumped single-mode MD laser that was monolithically grown on $\mathrm{Si}(001)$ substrate with $4^{\circ}$ offcut to the (011) plane. The laser structure, CW lasing emission spectra and threshold behavior are shown in Fig. 3d-f. By applying direct current injection, the minimum threshold current density of room-temperature-operated lasing was about $600 \mathrm{~A} \mathrm{~cm}^{-2}$. Using $\mathrm{V}$ grooved GaAs-on-Si template and V-groove (001) Si substrates, Wan et al. [36,37] further demonstrated the electrically pumped multi-mode QD MD lasing around $1.3 \mu \mathrm{m}$ with low power consumption and high temperature stability. These CW lasers can operate at high temperature up to $100^{\circ} \mathrm{C}$.

\section{COLLOIDAL NANOMATERIAL-BASED CW LASERS}

Although III-V semiconductor QD CW lasers have been intensively studied and gained great progress, the fabrication techniques, e.g., molecular beam epitaxy, CVD and liquid phase epitaxy, are expensive and complex. Moreover, it is difficult to realize the fabrication on flexible substrates. This drawback hinders the integration with flexible and low-cost photonic device.

Solution-processed high-gain media have been pursued 
a
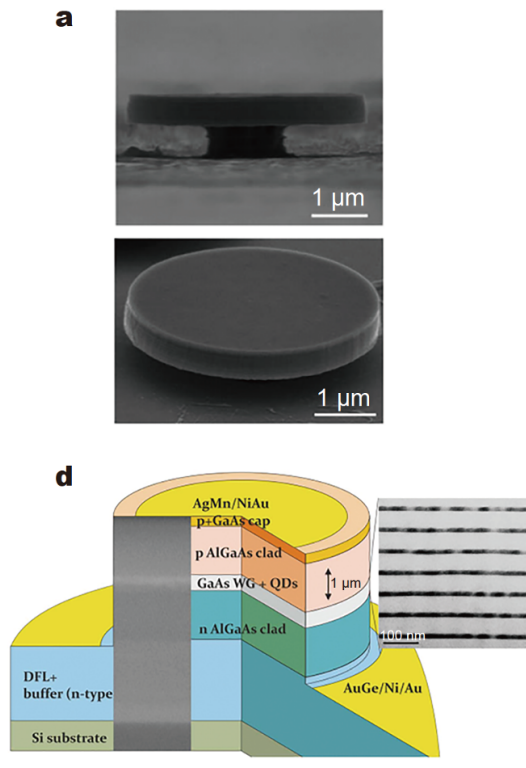

b
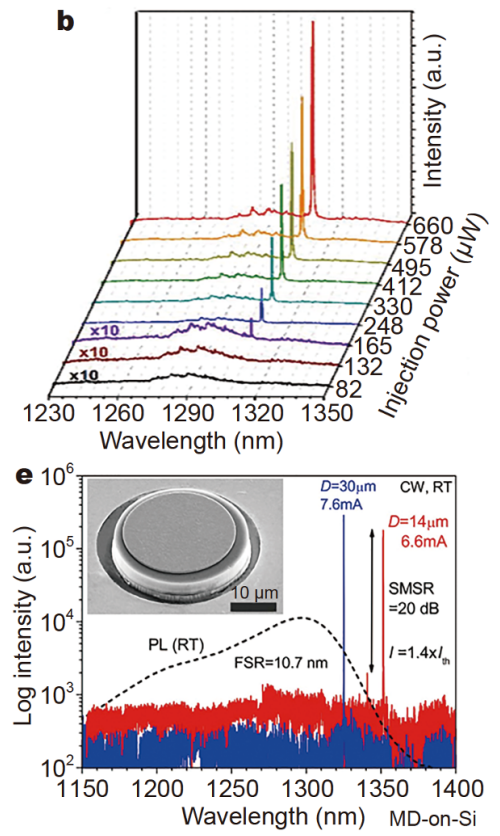

c
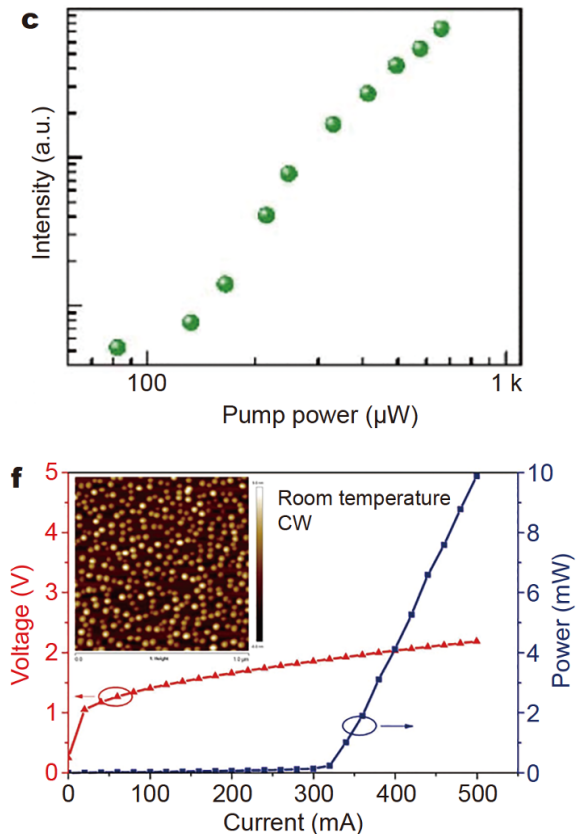

Figure 3 (a) SEM images of a $4-\mu \mathrm{m}$ fabricated MD with $90^{\circ}$ tilted view and $70^{\circ}$ tilted view. (b) Emission spectra and (c) integrated PL intensity of the dominant mode as a function of pump power. Reproduced with permission from [34]. Copyright 2016, The Optical Society (OSA). (d) Schematic of the layer sequence. The left inset shows a SEM image of the structure cross section; the right inset shows a bright-field TEM image of the QD active layers. (e) Room-temperature CW emission spectra, and (f) the threshold behavior. Reproduced with permission from [35]. Copyright 2017, The Optical Society (OSA).

for decades because of their easy synthesis and integration. CQDs are very competitive gain materials for solution-processed laser applications [15,38]. In contrast to the epitaxial III-V semiconductor QDs, CQDs have potential advantages of easy fabrication and integration. First, CQDs can be synthesized easily and inexpensively. Generally, CQDs are obtained through wet-chemical methods, e.g., hot-injection synthesis, one-pot non-injection organometallic synthesis, aqueous synthesis, and flow microreactor synthesis $[39,40]$. These methods do not rely on high-temperature, expensive vacuum-based processing and the expensive rigid substrates. Second, the high-quality CQDs have a high degree of monodispersity in solutions. They are compatible with almost all kinds of laser cavities. Third, the integration process of CQDs on $\mathrm{Si}$ or other platforms is swift and cost-effective. Using spin coating, Langmuir-Blodgett deposition or other nonvacuum techniques, we can transfer CQDs from a dispersion to a thin film easily [15]. Lastly, the absorption and the emission properties of CQDs can be tuned in the range from visible to infrared according to the composition and size control.

In comparison with the excellent performances of electrically pumped III-V QD-based CW lasers, solutionprocessed CQD CW lasers are still in their preliminary stage currently. There is no report on electrically pumped CQD CW lasers. The optically pumped CQD CW laser is a significant step toward reducing the threshold for integrated photonics [39].

\section{Challenges for CQD CW lasers}

The main obstacles for CQD achieving CW lasing emission are the large Auger recombination, the heat effect, and the poor uniformity of QD films. The Auger recombination is the most important factor affecting QD lasing threshold. Because of the non-unity degeneracy of band-edge states, the optical gain normally requires excitation of more than one exciton in each QD. However, the multiple excitons undergo rapid, nonradiative Auger recombination, prohibiting the sustained population inversion [41]. Another important factor is the heat effect. The optical performance of the QDs is temperature-dependent [42]. The high temperature will reduce the radiative efficiency, and increase the nonradiative Auger recombination $[43,44]$, following increasing the threshold of the amplified spontaneous emission (ASE) and lasing. The heat mainly comes out of two sources. One is the rapid light injection of the pump source, and the other is the quantum defect and nonradiative recombination of the QDs. The heat effect increases the nonradiative re- 
combination, which in turn generates more heat. What's more, the QD films normally suffer from low thermal conductivity. It accelerates the accumulation of heat in the QD films. The third factor is the poor uniformity of QD films on the laser cavities $[45,46]$. The rough surface of QD films causes much light scattering. It will consume the excited photons and produce more heat, resulting in the increase of the lasing threshold.

To reduce the lasing threshold and achieve CW lasing in CQDs, we need to decrease the defects of QDs, so as to suppress Auger recombination and improve the quantum yield (QY) of QDs. The elongated nanocrystals and heteronanocrystals can suppress the Auger recombination rates [47]. Several studies have achieved the quasi-CW and CW lasing in CdSe-based core/shell CQDs and 2D nanoplatelets $[42,45,47]$.

\section{CdSe and HgTe CQD-based quasi-CW or CW lasers}

Table 2 shows the major findings and key parameters of CdSe and HgTe CQD-based lasers. The investigation of CQDs for lasing application originates from Klimov's work in 2000 [48]. They proposed that the optical gain in CQDs did not rely on single-exciton emission, but on biexciton or mutiexcion emission. To illustrate this point, they modeled the band-edge emission in QDs using a two-level system with twofold spin-degenerate states and showed that the population inversion was due to two electron-hole pair states. The fast Auger recombination was the major obstacle preventing optical gain in CQDs. Klimov et al. [48] partially resolved this problem by employing close-packed QD films as the gain media and using femtosecond pulsed laser as the excitation source. The two experimental designs guaranteed the first observation of ASE in CQDs. After this work, the use of CQDs as gain medium for lasing has been widely studied. For example, the CQD lasers based on WG, distributed feedback (DFB) and random cavity were demonstrated [49-52].

Based on the optical gain models, the population inversion in CQDs can only be achieved if the average number of excitons is greater than one [48]. In 2004, Ivanov et al. [53] further developed a model to achieve optical gain with single exciton, and they successfully demonstrated the single exciton ASE from specially designed type-II CdS/ZnSe CQD later [54]. The type-II $\mathrm{CdS} / \mathrm{ZnSe}$ CQDs can spatially separate electrons and holes between the core and the shell, inducing a positive biexciton binding energy and shifting the absorption spectrum with respect to the luminescence line of CQDs. Five years later, Dang et al. [55] demonstrated that the single exciton gain could also be realized in pyramidal shaped type-I CdSe/ZnCdS core/shell CQDs.

It took more than fifteen years to achieve the optical pumped CQD-based CW laser. In 2013, using the type-I CQDs with CdSe core and thin ternary $\mathrm{Zn}_{0.5} \mathrm{Cd}_{0.5} \mathrm{~S}$ shell as gain media, Dang et al. [56] first demonstrated the single

Table 2 Representative milestones of colloidal CdSe and HgTe-based quasi-CW or CW lasers

\begin{tabular}{|c|c|c|c|c|c|c|}
\hline Authors & Materials & Type & Color & Thresholds & $\begin{array}{l}\text { Operation } \\
\text { condition }\end{array}$ & Publication year \\
\hline \multicolumn{7}{|l|}{ Pulse lasers } \\
\hline Klimov et al. [48] & CdSe QDs & ASE & Red & $\sim 8 \mathrm{~mW}$ & $80 \mathrm{~K}$ & 2000 \\
\hline Eisler et al. [50] & CdSe, CdSe(ZnS)QDs & Laser & Red & $\sim 0.9 \mu \mathrm{J}(80 \mathrm{~K})$ & $\mathrm{RT}$ and $80 \mathrm{~K}$ & 2002 \\
\hline Klimov et al. [54] & CdS/ZnSe QDs & ASE & Red & $2 \mathrm{~mJ} \mathrm{~cm}^{-2}$ & $\mathrm{RT}$ & 2007 \\
\hline Dang et al. [55] & CdSe/ZnCdS QDs & Laser & Red & $\sim 60 \mu \mathrm{J} \mathrm{cm}^{-2}$ & RT & 2012 \\
\hline \multicolumn{7}{|l|}{ Quasi-CW lasers } \\
\hline Dang et al. [56] & $\mathrm{CdSe} / \mathrm{Zn}_{0.5} \mathrm{Cd}_{0.5} \mathrm{~S}$ & Laser & Red & $250 \mu \mathrm{J} \mathrm{cm}^{-2}$ & & 2013 \\
\hline Wang et al. [57] & $\mathrm{CdZnS} / \mathrm{ZnS}$ & Laser & Blue & $30.2 \mu \mathrm{J}$ per pulse & RT & 2015 \\
\hline Adachi et al. [42] & CdSe/CdS/ZnS QDs & Laser & Red & $52 \mathrm{~kW} \mathrm{~cm}^{-2}$ & RT & 2015 \\
\hline \multicolumn{7}{|l|}{$\mathrm{CW}$ lasers } \\
\hline Fan et al. [47] & CdSe/CdS CQDs & Laser & Red & $6.4-8.4 \mathrm{~kW} \mathrm{~cm}^{-2}$ & $253 \mathrm{~K}$ & 2017 \\
\hline Yang et al. [45] & CdSe/CdS nanoplatelets & Laser & Red & $220 \mathrm{~W} \mathrm{~cm}^{-2}$ & RT & 2017 \\
\hline Wu et al. [60] & Charged CdSe/CdSe $\mathrm{S}_{1-x} / \mathrm{CdS}$ QDs & ASE & Red & $\begin{array}{c}500 \mathrm{~W} \mathrm{~cm}^{-2} \\
\text { (calculated value) }\end{array}$ & $\mathrm{RT}$ & 2017 \\
\hline Geiregat et al. [61] & HgTe QDs & ASE & Infrared & $40 \mathrm{~mW} \mathrm{~cm}^{-2}$ & $\mathrm{RT}$ & 2017 \\
\hline
\end{tabular}


mode lasers operated in quasi-CW regime. The laser was pumped by a solid-state laser with a pulse duration of 270 ps. It was 2.25 times longer than the multiexciton Auger recombination lifetime of the $\mathrm{CdSe} / \mathrm{Zn}_{0.5} \mathrm{Cd}_{0.5} \mathrm{~S}$ QDs, thus entering a quasi-steady state regime. The threshold of single mode lasing emission was about $250 \mu \mathrm{J} \mathrm{cm}^{-2}$. This achievement was mostly attributed to the high-quality densely packed CQD films. Later on, Sun's group from Nanyang Technological University [57] demonstrated quasi-CW blue liquid lasers from the solution of CdZnS/ZnS alloyed core/shell QDs. The pulse width of the pump laser used in this work was $5 \mathrm{~ns}$, which was one order of magnitude longer than the biexcitonic Auger recombination time constant $(\sim 350 \mathrm{ps})$ of the CdZnS/ZnS QDs.

In 2015, Sargent's group [42] realized that the main cause of the nanosecond-only operation of CQD lasers was thermal runaway. This can be attributed to the combination of rapid heat injection from the pump laser, poor heat removal and the temperature-dependent threshold. Using the ultra-compact CdSe/CdS/ZnS core/ shell/shell CQD film as gain media and the thermally conductive $\mathrm{MgF}_{2}$ material as substrate, they achieved microsecond-sustained CQD laser with a threshold of $52 \mathrm{~kW} \mathrm{~cm}^{-2}$. To further decrease the band-edge degeneracy and the single-dot photoluminescence (PL) linewidth of QDs, they created asymmetric CdSe/CdS core/ shell CQDs [47]. According to their method, CdS shell was grown on the (0001) facet of CdSe core, producing an asymmetric compressive shell in an oblate shape that created built-in biaxial strain, while still maintaining good surface passivation. The excitonic fine structure in biaxially strained dots could be spread uniformly and the thermal depopulation of the valence-band-edge was prevented. By incorporating the asymmetric biaxially strained CQDs film into a photonic crystal DFB cavity, they realized the $\mathrm{CW}$ lasing emission with a threshold of about $6.4-8.4 \mathrm{~kW} \mathrm{~cm}^{-2}$ with linewidth of about $3 \mathrm{meV}$. High-quality MD provides strong carrier confinement and high-quality-factor WG modes. Chien et al. [58] introduced $\mathrm{a} \mathrm{SiO}_{2}$ sandwiched colloidal CdSe/ZnS QD MD laser structure. The sandwich structure not only increased the confinement factor but also reduced the photo-oxidation of QDs under intense optical extraction. Based on this laser structure, they realized $\mathrm{CW}$ lasing with a threshold of $500 \mathrm{~kW} \mathrm{~cm}^{-2}$ and narrow linewidth of $0.18 \mathrm{~nm}$.

The 2D colloidal QWs (CQWs) hold several properties to achieve low threshold lasing. Firstly, the elongated nanocrystals can suppress Auger recombination due to the energy levels quantized in only one direction. It imposes a strict momentum conservation rule for Auger recombination. Secondly, the CQWs have larger spontaneous and stimulated emission cross sections. Thirdly, the loss due to sample heterogeneity can be largely avoided. In 2014, Grim et al. [41] fabricated 2D CdSe CQWs and showed that these CQWs possessed large exciton and biexciton binding energies, giving rise to room-temperature-operated CW laser. The threshold of the lasing was about $440 \mathrm{~W} \mathrm{~cm}^{-2}$. Using colloidal CdSe/ CdS core/shell nanoplatelets as gain media, Yang et al. [45] demonstrated a room-temperature-operated CW nanolaser with a threshold input power of less than $1 \mu \mathrm{W}$.

As of now, the CQD CW lasers are still at the laboratory stage. One of the most important challenges is the difficulty to realize the electrical pumped lasing. A key obstacle to realize that is the large Auger decay. The thick-shell with a continuously graded shell of QDs can suppress the Auger decay and preserve the strong confinement effect. Lim et al. [59] fabricated these thickshell, type-I CdSe/Cd $\mathrm{Zn}_{1-x} \mathrm{Se}$ QDs (Fig. 4a). In these nanocrystals, the confinement potentials are 'smoothened' for both electrons and holes, thus suppressing the hole- and electron-related Auger pathways. By applying a current-focusing device (Fig. 4b), which allows one to obtain a high current density up to $\sim 18 \mathrm{~A} \mathrm{~cm}^{-2}$ without damaging the QDs and the injection layers, they obtained direct-current electrical pumped optical gain with a threshold of 3-4 $\mathrm{A} \mathrm{cm}^{-2}$ (Fig. 4c).

To achieve population inversion, multi-excitons are normally required because of the non-unity degeneracy of the lowest-energy conduction band and valence band levels of QDs. It indicates that an intense pump pulse or high-power CW excitation is necessary to achieve lasing threshold. In 2017, Wu et al. [60] proposed a charged CQD concept to overcome this fundamental problem. By employing negatively charged QDs, the optical gain threshold can be reduced to sub-single-exciton or even zero-threshold level. Fig. 5a depicts the concept of zerothreshold optical gain, assuming the twofold degeneracy of the band-edge levels [60]. In neutral QDs, the single exciton state is optically transparent because the stimulated emission is exactly compensated by electron absorption. When the dot is occupied by an electron permanently, the ground-state absorption is reduced. A single-exciton excitation can produce optical gain. When a QD is doped with two or more electrons, the groundstate absorption can be completely bleached. 'Zerothreshold' gain can be achieved. Based on this idea, they realized the optical gain at extremely low excitation levels 

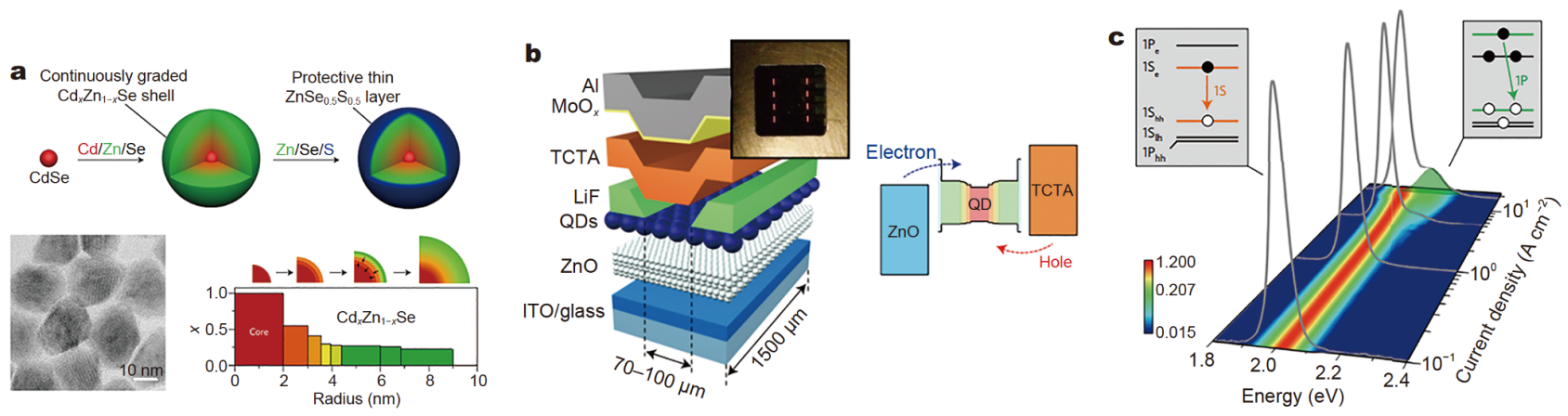

Figure 4 (a) Continuously graded core/shell CdSe/ $\mathrm{Cd}_{x} \mathrm{Zn}_{1-x} \mathrm{Se}_{\mathrm{ZnSe}} \mathrm{ZnS}_{0.5} \mathrm{~S}_{0.5}$ QDs (cg-QDs). (b) The light-emitting diode design used in this work features a tapered hole-injecting layer with a small-area contact $(70-100-\mu \mathrm{m}$ wide) to the cg-QD layer which allows boosting of the current density up to $18 \mathrm{~A} \mathrm{~cm}^{-2}$. A photograph of a real-life 8-pixel device under applied bias is shown as an inset. TCTA: tris(4-carbazoyl-9-ylphenyl)mine; ITO: indium tin oxide. (c) A color plot of electroluminescence (EL) spectra as a function of current density. At low currents, the device shows usual single-band EL due to the band-edge $1 \mathrm{~S}$ transition (orange states in the left inset). When the current density exceeds $\sim 1 \mathrm{~A} \mathrm{~cm}^{-2}$, the second higher-energy band $(\sim 2.15 \mathrm{eV})$ due to $1 \mathrm{P}$ transition (green states in the right inset) emerges in the EL spectra (green shading). Reproduced with permission from [59]. Copyright 2017, Springer Nature.

(Fig. $5 b$ and c).

Apart from the visible region, the CQD CW laser at the NIR telecom window is also very attractive because it can be applied in Si photonics directly. Geiregat et al. [61] first reported the infrared CW ASE with ultralow threshold of $40 \mathrm{~mW} \mathrm{~cm}^{-2}$ from colloidal HgTe QDs. The average excitation density $\langle N\rangle$ of the threshold value was as low as 0.005 per QD. The low gain threshold was attributed to the stimulated emission between the lowest conduction-band and an empty state in the bandgap of the HgTe QDs. Their results propose an approach for low threshold QD CW lasers based on intentional trap states.

\section{Perovskite-based quasi-CW or $\mathrm{CW}$ lasers}

During the last few years, colloidal metal halide perovskite semiconductors have become a hot research field in light sources owing to their superior properties such as easy fabrication process, tunable PL wavelength and narrow band emission. Metal halide perovskites have general chemical formula of $\mathrm{ABX}_{3}, \mathrm{~A}_{2} \mathrm{BX}_{5}$ or $\mathrm{A}_{4} \mathrm{BX}_{6}$, where $\mathrm{A}$ is $\mathrm{Cs}, \mathrm{CH}_{3} \mathrm{NH}_{3}$ (MA), $\mathrm{NH}_{2}-\mathrm{CH}=\mathrm{NH}_{2}$ (FA), etc., $\mathrm{B}$ is $\mathrm{Pb}, \mathrm{Sn}, \mathrm{Ge}$, etc., and $\mathrm{X}$ is $\mathrm{Cl}, \mathrm{Br}, \mathrm{I}[17,18,62,63]$. $2 \mathrm{D}$ Ruddlesden-Popper perovskite QWs with a chemical form of $\mathrm{L}_{2} \mathrm{~A}_{n-1} \mathrm{~B}_{n} \mathrm{X}_{3 n+1}$ can also be formed. Here $\mathrm{L}$ is large long organic chains that act as spacer between $\mathrm{BX}_{6}^{4-}$ octahedral layers and $n$ is the number of these layers. Perovskite nanomaterials are a class of excellent robust gain materials with high absorption coefficient and low density of defects [18,64-67]. They renew hope for a solution-processed CW lasing and set up a worldwide race to this goal.

Table 3 shows the major findings and key parameters of perovskite-based lasers, especially the quasi-CW or CW lasers. In 2014, Sum's group from Nanyang Technological University [68] demonstrated optical gain from $\mathrm{MAPbX}_{3}$ perovskite thin films for the first time. The wide wavelength tunable ASE with low threshold was observed at low temperature. Later on, using distributed Bragg reflector as Fabry-Pérot cavities, Deschler et al. [69] demonstrated the optically pumped room-temperatureoperated laser from solution-processed mixed halide perovskite semiconductors. From then on, the tunable ASE/lasing emission covering the entire visible range and NIR in perovskite nanowires (NWs), QDs or QWs are rapidly developed [16-18,39,65-67,70-97].

In 2015, Cadelano et al. [43] systematically discussed the conditions needed for realizing the true $\mathrm{CW}$ lasing form perovskite polycrystalline films. In this work, they first measured the ASE threshold of $\mathrm{MAPbI}_{3}$ and $\mathrm{MAPbBr}_{3}$ films as a function of temperature. They found that the threshold density was quadratically decreased with decrease of temperature, which was due to the temperature dependence of the radiative recombination rate and loss rate. They explored the ASE emission by varying the duration of the pump pulse from 4 to $300 \mathrm{~ns}$, and indicated that the runaway heating limited the longduration of ASE in perovskite films. The feasible improvements in heat management would allow the ASE to be sustained continuously at room temperature. Later, Jia et al. [98] proposed that the lasing duration of perovskite polycrystalline films were not just limited by thermal runaway. The lasing ceased mainly due to the photoinduced structural change in the perovskite. In the report, they fabricated high thermal conductivity Au/Si gratings. 

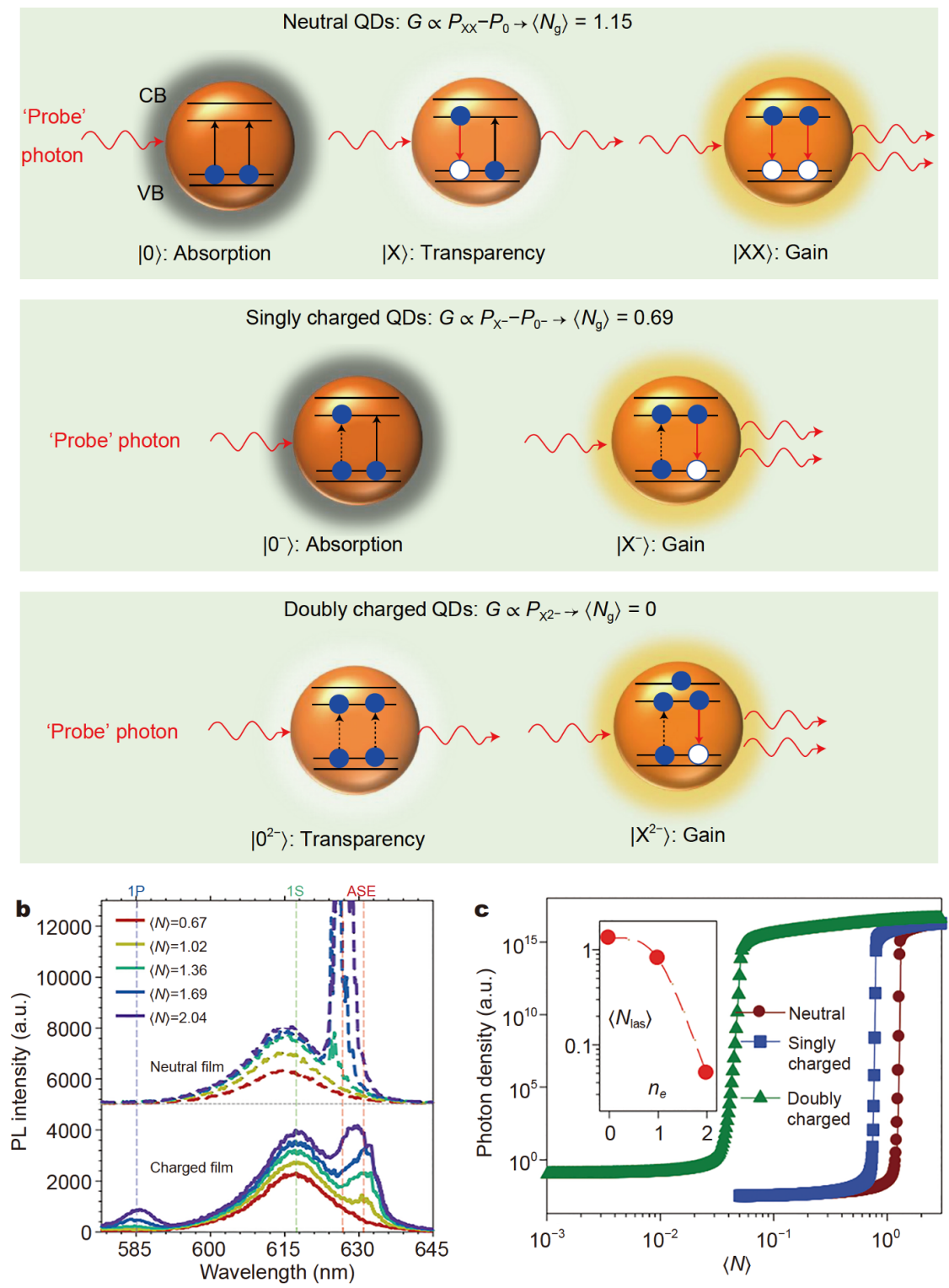

Figure 5 (a) Concept of zero-threshold optical gain. In a neutral QD with twofold spin-degenerate band-edge states. The gain threshold corresponds to $\langle N\rangle=1.15$. In QDs with one extra electron, the gain threshold is reduced to $\langle N\rangle=0.69$. In QDs doped with two extra electrons, the ground-state absorption is completely bleached, implying that optical gain is threshold-less. Filled and open blue circles are electrons and holes, respectively. Black and red arrows correspond to absorption and stimulated emission, respectively. Red wavy arrows are incoming and outgoing photons. (b) Pumpfluence-dependent PL spectra of the QD film before and after photochemical charging $\left(\left\langle n_{\mathrm{e}}\right\rangle=1.1\right)$ with $\langle N\rangle=0.67,1.02,1.36,1.69$ and 2.04 . (c) Modelling of time-integrated photon densities $(\Phi)$ in the cavity as a function of $\langle N\rangle$ for neutral, singly charged and doubly charged QDs. Reproduced with permission from [60]. Copyright 2017, Springer Nature.

Optically pumping the device with an InGaN diode laser at $160 \mathrm{~K}$, they achieved the quasi-CW lasing emission with a threshold of $5 \mathrm{~kW} \mathrm{~cm}^{-2}$ and duration of $\sim 25 \mathrm{~ns}$.

The first demonstration of CW lasing from perovskite was made in NWs, where lasing was attributed to polariton lasers. Polariton laser is one type of laser source whose coherent light emission is from the Bose-Einstein condensation of exciton-polaritons in semiconductors $[99,100]$. A polariton laser can emit coherent light at ultra-low pump power below the threshold for population inversion. In 2017, Evans et al. [101] reported the low temperature $(77 \mathrm{~K})$ operated $\mathrm{CW}$ polariton laser from $\mathrm{CsPbBr}_{3}$ perovskite NWs at excitation density of $6 \mathrm{~kW} \mathrm{~cm}^{-2}$. According to analysis of the cavity modes and 
Table 3 Representative milestones of perovskite-based quasi-CW or CW lasers

\begin{tabular}{|c|c|c|c|c|c|c|}
\hline Authors & Materials & Type & Color & Thresholds & $\begin{array}{l}\text { Operation } \\
\text { condition }\end{array}$ & Publication year \\
\hline \multicolumn{7}{|l|}{ Pulse lasers } \\
\hline Xing et al. [68] & $\mathrm{CH}_{3} \mathrm{NH}_{3} \mathrm{PbX}_{3}{ }^{\mathrm{a}}$ film & ASE & Visible & $\sim 10 \mu \mathrm{J} \mathrm{\textrm {cm } ^ { - 2 }}$ & RT & 2014 \\
\hline Deschler et al. [69] & $\mathrm{CH}_{3} \mathrm{NH}_{3} \mathrm{PbI}_{3-x} \mathrm{Cl}_{x}$ film & Laser & Red & $\sim 0.2 \mu \mathrm{J}$ per pulse & RT & 2014 \\
\hline Xing et al.[67] & $\mathrm{CH}_{3} \mathrm{NH}_{3} \mathrm{PbX}_{3} \mathrm{NWs}$ & Laser & Visible, near-infrared & $11 \mu \mathrm{J} \mathrm{cm}^{-2}$ & RT & 2015 \\
\hline Yakunin et al. [65] & $\mathrm{CsPbX}_{3}$ QDs & ASE/Laser & Visible & $\sim 5 \mu \mathrm{J} \mathrm{cm}{ }^{-2}$ & RT & 2015 \\
\hline \multicolumn{7}{|l|}{ Quasi-CW lasers } \\
\hline Cadelano et al. [43] & $\mathrm{MAPbX}_{3}$ film & ASE & Green, red & & $220 \mathrm{~K}$ & 2015 \\
\hline Jia et al. [98] & $\mathrm{MAPbI}_{3}$ film & Laser & Red & $5 \mathrm{~kW} \mathrm{~cm}^{-2}$ & $160 \mathrm{~K}$ & 2016 \\
\hline \multicolumn{7}{|l|}{ CW lasers } \\
\hline Evans et al. [101] & $\mathrm{CsPbBr}_{3} \mathrm{NWs}$ & Laser & Green & $6 \mathrm{~kW} \mathrm{~cm}^{-2}$ & $77 \mathrm{~K}$ & 2017 \\
\hline Jia et al. [103] & $\mathrm{MAPbI}_{3}$ film & Laser & Red & $\sim 17 \mathrm{~kW} \mathrm{~cm}^{-2}$ & $102 \mathrm{~K}$ & 2017 \\
\hline Li et al. [104] & $\mathrm{MAPbI}_{3}$ film & Laser & Red & $13 \mathrm{~W} \mathrm{~cm}^{-2}$ & RT & 2018 \\
\hline Brenner et al.[105] & $\begin{array}{c}\mathrm{Cs}_{0.1}\left(\mathrm{MA}_{0.17} \mathrm{FA}_{0.83}\right)_{0.9} \mathrm{~Pb}_{0.84^{-}} \\
\left(\mathrm{I}_{0.84} \mathrm{Br}_{0.16}\right)_{2.68} \text { film }\end{array}$ & ASE & Red & $387 \mathrm{~W} \mathrm{~cm}^{-2}$ & $80 \mathrm{~K}$ & 2019 \\
\hline Wang et al. [106] & $\mathrm{MAPbX}_{3}$ QDs & Laser & Green, blue, red & $\begin{array}{l}\text { Green: } 15 \mathrm{~W} \mathrm{~cm}^{-2} \\
\text { Red: } 24 \mathrm{~W} \mathrm{~cm}^{-2} \\
\text { Blue: } 58 \mathrm{~W} \mathrm{~cm}^{-2}\end{array}$ & RT & 2019 \\
\hline
\end{tabular}

a) $\mathrm{X}$ represents $\mathrm{Cl}, \mathrm{Br}, \mathrm{I}$.

their temperature dependence, they revealed that the CW lasing originated from polariton modes near the bottleneck region on the lower polariton branch. This work indicates that perovskite NWs are promising materials for polariton lasers. Later on, Jiang et al. [102] further reduced the linewidth of $\mathrm{CsPbX}_{3} \mathrm{NW} \mathrm{CW}$ nanolasers to $0.09 \mathrm{~nm}$ in low temperature condition.

The first report on the real photon CW lasers was from Giebink's group [103]. They fabricated a DFB laser structure by depositing a $\sim 120$-nm-thick $\mathrm{MAPbI}_{3}$ polycrystalline film on the alumina grating that lied on a highthermal-conductivity sapphire substrate. The lasing structure, lasing emission and lasing threshold behavior are shown in Fig. 6a-c. At temperatures below its tetragonal-to-orthorhombic phase transition, obviously polarized lasing emission with a threshold of $\sim 17 \mathrm{~kW} \mathrm{~cm}^{-2}$ was observed. The continuous gain originates from tetragonal-phase inclusions that are photogenerated by the pump within the bulk orthorhombic host matrix. Li et al. [104] directly patterned DFB gratings on $\mathrm{MAPbI}_{3}$ polycrystalline films using thermal nanoimprint lithography and raised the operation temperature of the $\mathrm{CW}$ laser to room-temperature. Fig. $6 \mathrm{~d}$ shows the schematic illustration of the nanoimprint process of the imprinted $\mathrm{MAPbI}_{3}$ DFB resonator. The direct patterning of perovskite not only provided a high-quality-factor cavity with large mode-gain overlap but also improved emission characteristics of the perovskite films. The ultra-low lasing threshold of $13 \mathrm{~W} \mathrm{~cm}^{-2}$ was achieved, just as shown in Fig. 6e and f. Brenner et al. [105] employed a phase-stable perovskite film by spin-casting a precursor solution containing triple cation system with a non-stoichiometric ratio of $\mathrm{Cs}_{0.1}\left(\mathrm{MA}_{0.17} \mathrm{FA}_{0.83}\right)_{0.9} \mathrm{~Pb}_{0.84}\left(\mathrm{I}_{0.84} \mathrm{Br}_{0.16}\right)_{2.68}$, and demonstrated CW ASE at temperatures from 80 to $120 \mathrm{~K}$. They also studied the temperature-dependent ASE properties and showed that the material degradation prohibited the ASE at high temperatures.

All the perovskite CW lasers mentioned above are based on perovskite NWs or polycrystalline films. These materials still need to be rationally designed toward easy integration and large-scale fabrication. Perovskite QDs (PQDs) have better optical performance compared with the NWs and polycrystalline films [20,64]. Moreover, the PQDs can be in situ fabricated in polymeric matrix, which provides highly luminescent composite films with advantageous features of easy fabrication and integration. Very recently, by carefully choosing polyacrylonitrile (PAN) as polymeric matrix, our group obtained in situ fabricated PQD-embedded PAN films and realized ultralow-threshold and color-tunable PQD-based CW lasers operated at room-temperature for the first time [106]. The PQD-embedded PAN films had outstanding 

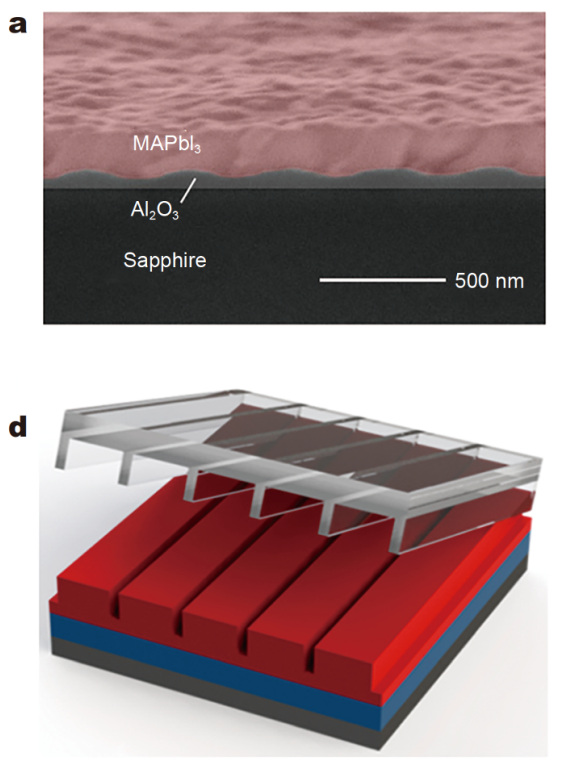

b
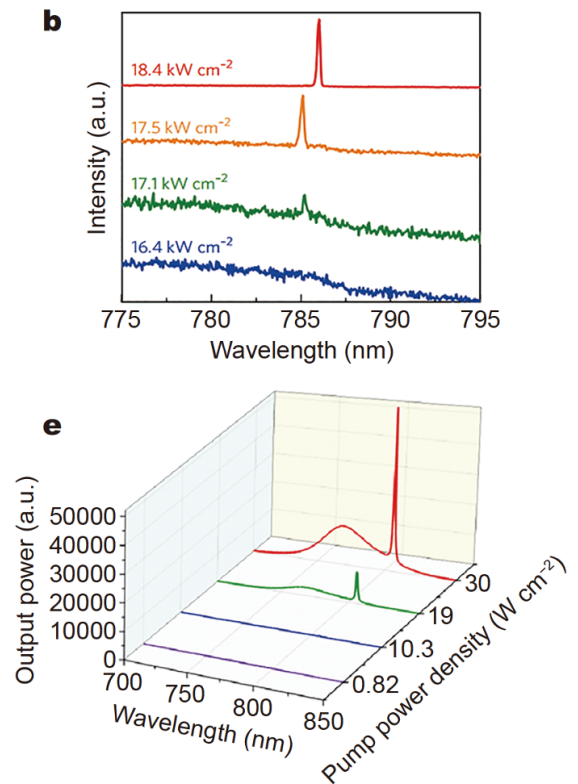

C
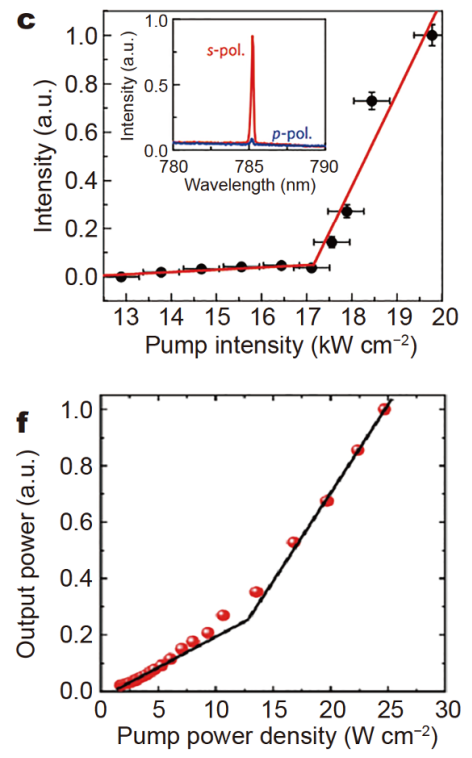

Figure 6 (a) Cross-sectional SEM of the second-order DFB laser architecture consisting of a MAPbI ${ }_{3}$ film deposited on an 80-nm-thick etched alumina grating on a sapphire substrate. (b) The emission spectra and (c) input-output characteristics of the laser under different continuous excitation powers at $102 \mathrm{~K}$. The emission spectrum above threshold is strongly s-polarized. Reproduced with permission from [103]. Copyright 2017,

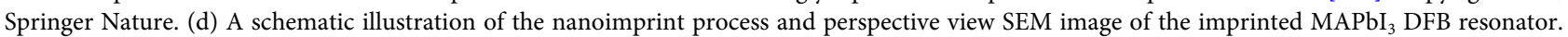
(e) CW lasing characteristics at room-temperature and (f) lasing threshold behavior. Reproduced with permission from [104]. Copyright 2018, American Chemical Society.

optical and thermal properties. They had high transmittance ( $90 \%$ ), near-unity PLQYs (up to $97 \%$ ), very smooth surface (root-mean-square roughness: $0.784 \mathrm{~nm}$ ), and high thermal conductivity $\left(\sim 1.2 \mathrm{~W} \mathrm{~m}^{-1} \mathrm{~K}^{-1}\right)$ (Fig. 7ac). Moreover, the PQDs show unique features of charge spatial separation effects due to the interactions between the in situ fabricated PQDs and the PAN matrix. Combining those films with DFB cavities, we obtained green, blue and red room-temperature-operated CW lasers with thresholds as low as 15,58 , and $24 \mathrm{~W} \mathrm{~cm}^{-2}$, respectively (Fig. 7d-f). This progress opens up a door to achieve perovskite CW lasers for photonic application.

Although perovskite-based lasers have made great progress and several groups have achieved CW laser, it remains an open question whether perovskites are excellent gain materials to achieve room-temperature-operated CW lasing. A recent comment by Brenner et al. [107] expressed their reservations about the validity of room-temperature CW laser.

\section{CONCLUSION AND OUTLOOK}

In this work, we review the recent developments of QDbased CW lasers for photonic applications. The QDs can be classified into two categories: the epitaxial QDs and the solution-processed CQDs. We start with the summary of epitaxial III-V semiconductor QD-based CW lasers on $\mathrm{Ge}, \mathrm{Ge}-\mathrm{on}-\mathrm{Si}$ and $\mathrm{Si}$ substrates, and then discuss the difficulties and challenges associated with the realization of room-temperature-operated CQD CW lasers. In the last part, we also review the progress toward perovskite nanocrystal-based CW lasers.

To meet the commercial on-chip application, CW lasers with high performance, easy integration, and low cost are expected. Epitaxial III-V QD-based CW lasers, to some extent, are a promising candidate as on-chip light source. They are compatible with the semiconductor fabrication infrastructure and can realize electrical pump easily. Owing to the huge effort, the electrically pumped epitaxial QD CW lasers on Si substrates have achieved great advance. The CW lasing emission with low threshold of $62.5 \mathrm{~A} \mathrm{~cm}^{-2}$, high output power of $>105 \mathrm{~mW}$, long lifetime operation of $3100 \mathrm{~h}$ and high operation temperature to $100^{\circ} \mathrm{C}$ were achieved. However, a few roadblocks remain unresolved. One big challenge is the reduction of the defect density caused by the dissimilarity between III-V materials and Si substrates. Deep understanding about the growing process is still necessary to achieve precise control over the interface.

CQDs have good solution-processability and easy integration properties. The CQD CW lasers can provide a 

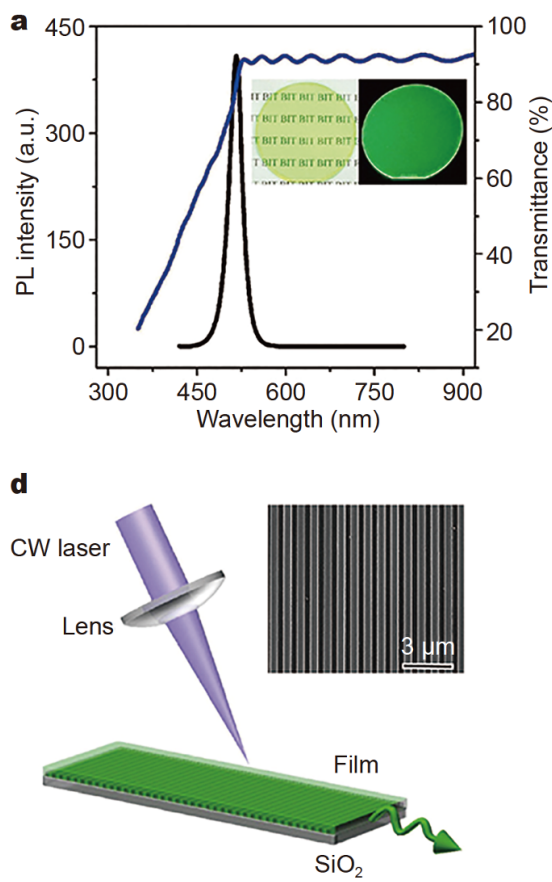
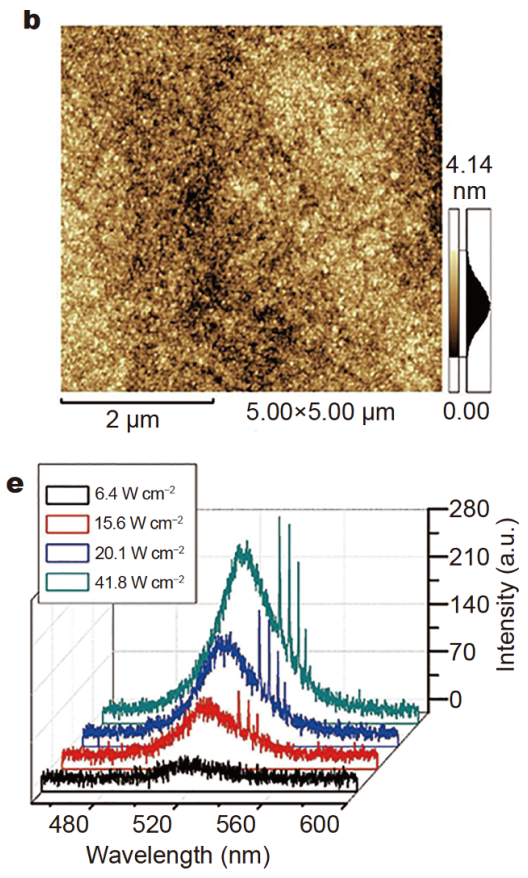

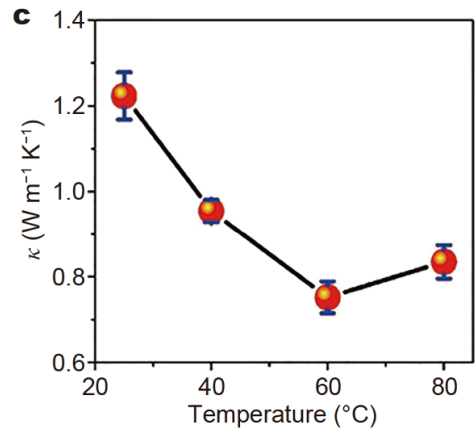

f

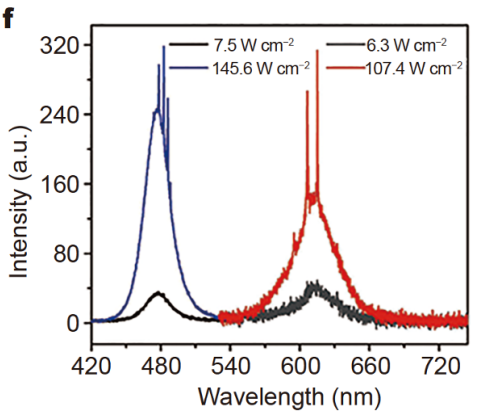

Figure 7 (a) Transmittance and PL spectra of the in situ fabricated MAPbBr ${ }_{3}$ PQD-embedded PAN film. The inset shows an optical image of the film under daylight and ultraviolet light. (b) $5 \mu \mathrm{m} \times 5 \mu \mathrm{m}$ AFM image of the 1.9- $\mathrm{mm}$-thick film spin-coated on quartz glass. The root-mean-square roughness of the film is $0.784 \mathrm{~nm}$. (c) Thermal conductivities of the film at different temperatures. (d) Schematic setup of the room-temperature CW lasing measurement. The inset shows an SEM image of the grating. (e) Emission spectra of the film on the DFB cavity. (f) Lasing emission spectra of the blue and red PQD-embedded PAN films on DFB cavities. Reproduced with permission from [106]. Copyright 2019, American Chemical Society.

promising "hybrid approach" that combines the CQD lasers directly with Si substrates. Besides, CQDs have advantages of tunable emission wavelengths and low fabrication cost, making them appealing for on-chip applications. However, compared with the advance of epitaxial QD CW lasers, the solution-processed CQD CW lasers are still in an initial stage, although mill-stonebreakthroughs were made recently.

The biggest barrier for CQD CW lasers is the high threshold. Up to date, the electrically pumped CQD CW lasing has not been reported. It is because the lasing threshold is too high and CQDs cannot withstand high current injection. Sustaining large optical gain under strong pumping power is another challenge. High output power and long lifetime are the requirements for commercial laser products. That means the CQDs must possess the capability of high-power and long-duration pumping. However, the optical gain of CQDs is extremely sensitive to the pump power and heat due to the temperature-dependent properties of Auger recombination [44]. The next challenge is the fabrication quality of CQD films. Currently, the CQDs in solution play a dominant role in photonic applications. Transferring CQDs from a dispersion to a high-quality film is not quite easy.

To overcome these barriers, the CQDs need more indepth research from fundamental chemical physics and preparation technology. Achieving optical gain down to sub-exciton, suppressing Auger recombination at high pump power, improving stability at high temperatures and high current injection require huge efforts.

Perovskites would probably make a breakthrough in the field of electrically pumped CQD lasers. Perovskite materials have excellent gain properties, such as high absorption coefficient and low density of defects [18]. A high absorption coefficient guarantees a large optical gain. It is reported that the net optical gain of $\mathrm{MAPbI}_{3}$ perovskite thin film is about $3200 \mathrm{~cm}^{-1}$, which is higher than that of traditional CQDs [108]. The optical loss, including phonon scattering and edge scattering, will consume the photons and increase the laser threshold. The low density of defects in perovskite is helpful to reduce the optical loss. Miao et al. [109] have proved that the perovskite-based light-emitting diodes are able to keep high external quantum efficiency at a high current density of $300 \mathrm{~mA} \mathrm{~cm}^{-2}$. This fact demonstrates that the perovskite materials have the ability to withstand high 
current densities. In addition, in situ fabricated PQDs provide an effective route to achieve high-quality QD film without using solution process. Very recently, the PQDsembedded glasses were fabricated by applying laser irradiation [110].

Although CQD-based CW lasers are in an initial stage, they show a remarkable potential to integrate into onchip photonics. With the understanding of the physics of gain building and annihilating, there are plenty of rooms to realize the required CW lasers. Maybe the answer is perovskites.

\section{Received 19 February 2020; accepted 6 April 2020; published online 26 May 2020}

1 Zhou Z, Yin B, Michel J. On-chip light sources for silicon photonics. Light Sci Appl, 2015, 4: e358

2 Paul DJ. Si/SiGe heterostructures: From material and physics to devices and circuits. Semicond Sci Technol, 2004, 19: R75-R108

3 Chen $\mathrm{S}, \mathrm{Li} \mathrm{W}, \mathrm{Wu} \mathrm{J}$, et al. Electrically pumped continuous-wave III-V quantum dot lasers on silicon. Nat Photon, 2016, 10: 307311

4 Dai D, Bauters J, Bowers JE. Passive technologies for future largescale photonic integrated circuits on silicon: polarization handling, light non-reciprocity and loss reduction. Light Sci Appl, 2012, 1: el

5 Thomson DJ, Gardes FY, Fedeli JM, et al. $50-\mathrm{GB} / \mathrm{s}$ silicon optical modulator. IEEE Photon Technol Lett, 2012, 24: 234-236

6 Vivien L, Polzer A, Marris-Morini D, et al. Zero-bias $40 \mathrm{Gbit} / \mathrm{s}$ germanium waveguide photodetector on silicon. Opt Express, 2012, 20: 1096-1101

7 Streshinsky M, Ding R, Liu Y, et al. Low power $50 \mathrm{~Gb} / \mathrm{s}$ silicon traveling wave mach-zehnder modulator near $1300 \mathrm{~nm}$. Opt Express, 2013, 21: 30350-30357

8 Thomson D, Zilkie A, Bowers JE, et al. Roadmap on silicon photonics. J Opt, 2016, 18: 073003

9 Liu AY, Peters J, Huang X, et al. Electrically pumped continuouswave $13 \mu \mathrm{m}$ quantum-dot lasers epitaxially grown on on-axis (001) GaP/Si. Opt Lett, 2017, 42: 338-341

10 Jung D, Norman J, Kennedy MJ, et al. High efficiency low threshold current $1.3 \mu \mathrm{m}$ InAs quantum dot lasers on on-axis (001) GaP/Si. Appl Phys Lett, 2017, 111: 122107

11 Jung D, Zhang Z, Norman J, et al. Highly reliable low-threshold InAs quantum dot lasers on on-axis (001) Si with $87 \%$ injection efficiency. ACS Photonics, 2018, 5: 1094-1100

12 Norman J, Kennedy MJ, Selvidge J, et al. Electrically pumped continuous wave quantum dot lasers epitaxially grown on patterned, on-axis (001) Si. Opt Express, 2017, 25: 3927-3934

13 Chen S, Liao M, Tang M, et al. Electrically pumped continuouswave $13 \mu \mathrm{m}$ InAs/GaAs quantum dot lasers monolithically grown on on-axis Si (001) substrates. Opt Express, 2017, 25: 4632-4639

14 Pan S, Cao V, Liao M, et al. Recent progress in epitaxial growth of III-V quantum-dot lasers on silicon substrate. J Semicond, 2019, 40: 101302

15 Xie W, Zhu Y, Bisschop S, et al. Colloidal quantum dots enabling coherent light sources for integrated silicon-nitride photonics. IEEE J Sel Top Quantum Electron, 2017, 23: 1-13 quantum dots: A new class of lasing materials with favorable characteristics. Adv Mater, 2015, 27: 7101-7108

17 Veldhuis SA, Boix PP, Yantara N, et al. Perovskite materials for light-emitting diodes and lasers. Adv Mater, 2016, 28: 6804-6834

18 Zhang Q, Su R, Du W, et al. Advances in small perovskite-based lasers. Small Methods, 2017, 1: 1700163

19 Liu Z, Li C, Shang QY, et al. Research progress of low-dimensional metal halide perovskites for lasing applications. Chin Phys B, 2018, 27: 114209

20 Chen J, Du W, Shi J, et al. Perovskite quantum dot lasers. InfoMat, 2020, 2: 170-183

21 Li C, Liu Z, Shang Q, et al. Surface-plasmon-assisted metal halide perovskite small lasers. Adv Opt Mater, 2019, 7: 1900279

22 Mi Y, Zhong Y, Zhang Q, et al. Continuous-wave pumped perovskite lasers. Adv Opt Mater, 2019, 7: 1900544

23 Wang K, Wang S, Xiao S, et al. Recent advances in perovskite micro- and nanolasers. Adv Opt Mater, 2018, 6: 1800278

24 Alferov ZI, Andreev VM, Garbuzov DZ, et al. Investigation of the influence of the AlAs-GaAs heterostructure parameters on the laser threshold current and the realization of continuous emission at room temperature. Sov Phys Semicond, 1971, 4: 1573-1575

25 Arakawa Y, Sakaki H. Multidimensional quantum well laser and temperature dependence of its threshold current. Appl Phys Lett, 1982, 40: 939-941

26 Kirstaedter N, Ledentsov NN, Grundmann $\mathrm{M}$, et al. Low threshold, large to injection laser emission from (InGa)As quantum dots. Electron Lett, 1994, 30: 1416-1417

27 Norman JC, Jung D, Wan Y, et al. Perspective: The future of quantum dot photonic integrated circuits. APL Photonics, 2018, 3: 030901

28 Kazi ZI, Egawa T, Jimbo T, et al. First room-temperature continuous-wave operation of self-formed InGaAs quantum dot-like laser on Si substrate grown by metalorganic chemical vapor deposition. Jpn J Appl Phys, 2000, 39: 3860-3862

29 Liu $\mathrm{H}$, Wang $\mathrm{T}$, Jiang $\mathrm{Q}$, et al. Long-wavelength InAs/GaAs quantum-dot laser diode monolithically grown on Ge substrate. Nat Photon, 2011, 5: 416-419

30 Lee A, Jiang Q, Tang $\mathrm{M}$, et al. Continuous-wave InAs/GaAs quantum-dot laser diodes monolithically grown on $\mathrm{Si}$ substrate with low threshold current densities. Opt Express, 2012, 20: 22181-22187

31 Liu AY, Zhang C, Norman J, et al. High performance continuous wave $1.3 \mu \mathrm{m}$ quantum dot lasers on silicon. Appl Phys Lett, 2014, 104: 041104

32 Liu AY, Herrick RW, Ueda O, et al. Reliability of InAs/GaAs quantum dot lasers epitaxially grown on silicon. IEEE J Sel Top Quantum Electron, 2015, 21: 690-697

33 Wang Y, Chen S, Yu Y, et al. Monolithic quantum-dot distributed feedback laser array on silicon. Optica, 2018, 5: 528

34 Wan Y, Li Q, Liu AY, et al. Optically pumped $13 \mu \mathrm{m}$ roomtemperature InAs quantum-dot micro-disk lasers directly grown on (001) silicon. Opt Lett, 2016, 41: 1664-1667

35 Kryzhanovskaya N, Moiseev E, Polubavkina Y, et al. Heat-sink free $\mathrm{CW}$ operation of injection microdisk lasers grown on $\mathrm{Si}$ substrate with emission wavelength beyond $13 \mu \mathrm{m}$. Opt Lett, 2017, 42: 3319

36 Wan Y, Norman J, Li Q, et al. $13 \mu \mathrm{m}$ submilliamp threshold quantum dot micro-lasers on Si. Optica, 2017, 4: 940

37 Wan Y, Jung D, Norman J, et al. O-band electrically injected quantum dot micro-ring lasers on on-axis (001) GaP/Si and V- 
groove Si. Opt Express, 2017, 25: 26853-26860

38 Pietryga JM, Park YS, Lim J, et al. Spectroscopic and device aspects of nanocrystal quantum dots. Chem Rev, 2016, 116: 1051310622

39 Wang Y, Sun H. Advances and prospects of lasers developed from colloidal semiconductor nanostructures. Prog Quantum Electron, 2018, 60: 1-29

$40 \mathrm{Pu} \mathrm{Y}$, Cai F, Wang D, et al. Colloidal synthesis of semiconductor quantum dots toward large-scale production: A review. Ind Eng Chem Res, 2018, 57: 1790-1802

41 Grim JQ, Christodoulou S, Di Stasio F, et al. Continuous-wave biexciton lasing at room temperature using solution-processed quantum wells. Nat Nanotech, 2014, 9: 891-895

42 Adachi MM, Fan F, Sellan DP, et al. Microsecond-sustained lasing from colloidal quantum dot solids. Nat Commun, 2015, 6: 8694

43 Cadelano M, Sarritzu V, Sestu N, et al. Can trihalide lead perovskites support continuous wave lasing? Adv Opt Mater, 2015, 3: 1557-1564

44 Javaux C, Mahler B, Dubertret B, et al. Thermal activation of nonradiative auger recombination in charged colloidal nanocrystals. Nat Nanotech, 2013, 8: 206-212

45 Yang Z, Pelton M, Fedin I, et al. A room temperature continuouswave nanolaser using colloidal quantum wells. Nat Commun, 2017, 8: 143

46 Gupta S, Waks E. Spontaneous emission enhancement and saturable absorption of colloidal quantum dots coupled to photonic crystal cavity. Opt Express, 2013, 21: 29612-29619

47 Fan F, Voznyy O, Sabatini RP, et al. Continuous-wave lasing in colloidal quantum dot solids enabled by facet-selective epitaxy. Nature, 2017, 544: 75-79

48 Klimov VI, Mikhailovsky AA, Xu S, et al. Optical gain and stimulated emission in nanocrystal quantum dots. Science, 2000, 290: 314-317

49 Klimov VI, Bawendi MG. Ultrafast carrier dynamics, optical amplification, and lasing in nanocrystal quantum dots. MRS Bull, 2001, 26: 998-1004

50 Eisler HJ, Sundar VC, Bawendi MG, et al. Color-selective semiconductor nanocrystal laser. Appl Phys Lett, 2002, 80: 4614-4616

51 Kazes M, Lewis DY, Ebenstein Y, et al. Lasing from semiconductor quantum rods in a cylindrical microcavity. Adv Mater, 2002, 14: 317-321

$52 \mathrm{Xu}$ J, Xiao M. Lasing action in colloidal CdS/CdSe/CdS quantum wells. Appl Phys Lett, 2005, 87: 173117

53 Ivanov SA, Nanda J, Piryatinski A, et al. Light amplification using inverted core/shell nanocrystals: towards lasing in the single-exciton regime. J Phys Chem B, 2004, 108: 10625-10630

54 Klimov VI, Ivanov SA, Nanda J, et al. Single-exciton optical gain in semiconductor nanocrystals. Nature, 2007, 447: 441-446

55 Dang C, Lee J, Breen C, et al. Red, green and blue lasing enabled by single-exciton gain in colloidal quantum dot films. Nat Nanotech, 2012, 7: 335-339

56 Dang C, Lee J, Roh K, et al. Highly efficient, spatially coherent distributed feedback lasers from dense colloidal quantum dot films. Appl Phys Lett, 2013, 103: 171104

57 Wang Y, Leck KS, Ta VD, et al. Blue liquid lasers from solution of $\mathrm{CdZnS/ZnS} \mathrm{ternary} \mathrm{alloy} \mathrm{quantum} \mathrm{dots} \mathrm{with} \mathrm{quasi-continuous}$ pumping. Adv Mater, 2015, 27: 169-175

58 Chien HC, Cheng CY, Mao MH. Continuous wave operation of $\mathrm{SiO}_{2}$ sandwiched colloidal $\mathrm{CdSe} / \mathrm{ZnS}$ quantum-dot microdisk la- sers. IEEE J Sel Top Quantum Electron, 2017, 23: 1-5

59 Lim J, Park YS, Klimov VI. Optical gain in colloidal quantum dots achieved with direct-current electrical pumping. Nat Mater, 2018, 17: $42-49$

60 Wu K, Park YS, Lim J, et al. Towards zero-threshold optical gain using charged semiconductor quantum dots. Nat Nanotech, 2017, 12: $1140-1147$

61 Geiregat P, Houtepen AJ, Sagar LK, et al. Continuous-wave infrared optical gain and amplified spontaneous emission at ultralow threshold by colloidal HgTe quantum dots. Nat Mater, 2018, 17: 35-42

62 Chang S, Bai Z, Zhong H. In situ fabricated perovskite nanocrystals: A revolution in optical materials. Adv Opt Mater, 2018, 6: 1800380

63 Wei Q, Li X, Liang C, et al. Recent progress in metal halide perovskite micro- and nanolasers. Adv Opt Mater, 2019, 7: 1900080

64 Zhang $\mathrm{F}$, Zhong $\mathrm{H}$, Chen $\mathrm{C}$, et al. Brightly luminescent and colortunable colloidal $\mathrm{CH}_{3} \mathrm{NH}_{3} \mathrm{PbX}_{3}(\mathrm{X}=\mathrm{Br}, \mathrm{I}, \mathrm{Cl})$ quantum dots: Potential alternatives for display technology. ACS Nano, 2015, 9: 4533-4542

65 Yakunin S, Protesescu L, Krieg F, et al. Low-threshold amplified spontaneous emission and lasing from colloidal nanocrystals of caesium lead halide perovskites. Nat Commun, 2015, 6: 8056

66 Zhang Q, Ha ST, Liu X, et al. Room-temperature near-infrared high-Q perovskite whispering-gallery planar nanolasers. Nano Lett, 2014, 14: 5995-6001

67 Xing J, Liu XF, Zhang Q, et al. Vapor phase synthesis of organometal halide perovskite nanowires for tunable room-temperature nanolasers. Nano Lett, 2015, 15: 4571-4577

68 Xing G, Mathews N, Lim SS, et al. Low-temperature solutionprocessed wavelength-tunable perovskites for lasing. Nat Mater, 2014, 13: 476-480

69 Deschler F, Price M, Pathak S, et al. High photoluminescence efficiency and optically pumped lasing in solution-processed mixed halide perovskite semiconductors. J Phys Chem Lett, 2014, 5: $1421-1426$

70 Sutherland BR, Hoogland S, Adachi MM, et al. Conformal organohalide perovskites enable lasing on spherical resonators. ACS Nano, 2014, 8: 10947-10952

71 Liao Q, Hu K, Zhang H, et al. Perovskite microdisk microlasers self-assembled from solution. Adv Mater, 2015, 27: 3405-3410

72 Zhu H, Fu Y, Meng F, et al. Lead halide perovskite nanowire lasers with low lasing thresholds and high quality factors. Nat Mater, 2015, 14: 636-642

73 Eaton SW, Lai M, Gibson NA, et al. Lasing in robust cesium lead halide perovskite nanowires. Proc Natl Acad Sci USA, 2016, 113 : 1993-1998

74 Fu Y, Zhu H, Stoumpos CC, et al. Broad wavelength tunable robust lasing from single-crystal nanowires of cesium lead halide perovskites $\left(\mathrm{CsPbX}_{3}, \mathrm{X}=\mathrm{Cl}, \mathrm{Br}, \mathrm{I}\right)$. ACS Nano, 2016, 10: 79637972

75 Li YJ, Lv Y, Zou CL, et al. Output coupling of perovskite lasers from embedded nanoscale plasmonic waveguides. J Am Chem Soc, 2016, 138: 2122-2125

76 Saliba M, Wood SM, Patel JB, et al. Structured organic-inorganic perovskite toward a distributed feedback laser. Adv Mater, 2016, 28: 923-929

77 Shi ZF, Sun XG, Wu D, et al. Near-infrared random lasing realized in a perovskite $\mathrm{CH}_{3} \mathrm{NH}_{3} \mathrm{PbI}_{3}$ thin film. J Mater Chem $\mathrm{C}$, 
2016, 4: 8373-8379

78 Tang $\mathrm{X}, \mathrm{Hu} \mathrm{Z}$, Chen $\mathrm{W}$, et al. Room temperature single-photon emission and lasing for all-inorganic colloidal perovskite quantum dots. Nano Energy, 2016, 28: 462-468

79 Wang X, Wang X, Pan A. Continuous-wave lasing in halide perovskites. Sci China Mater, 2018, 61: 1243-1244

$80 \mathrm{Xu} \mathrm{Y,} \mathrm{Chen} \mathrm{Q,} \mathrm{Zhang} \mathrm{C,} \mathrm{et} \mathrm{al.} \mathrm{Two-photon-pumped} \mathrm{perovskite}$ semiconductor nanocrystal lasers. J Am Chem Soc, 2016, 138: 3761-3768

81 Zhang Q, Su R, Liu X, et al. High-quality whispering-gallerymode lasing from cesium lead halide perovskite nanoplatelets. Adv Funct Mater, 2016, 26: 6238-6245

82 Zhang W, Peng L, Liu J, et al. Controlling the cavity structures of two-photon-pumped perovskite microlasers. Adv Mater, 2016, 28: 4040-4046

83 Huang CY, Zou C, Mao C, et al. $\mathrm{CsPbBr}_{3}$ perovskite quantum dot vertical cavity lasers with low threshold and high stability. ACS Photonics, 2017, 4: 2281-2289

84 Su R, Diederichs C, Wang J, et al. Room-temperature polariton lasing in all-inorganic perovskite nanoplatelets. Nano Lett, 2017, 17: 3982-3988

85 Wei Q, Du B, Wu B, et al. Two-photon optical properties in individual organic-inorganic perovskite microplates. Adv Opt Mater, 2017, 5: 1700809

86 Li M, Gao Q, Liu P, et al. Amplified spontaneous emission based on 2D Ruddlesden-Popper perovskites. Adv Funct Mater, 2018, 28: 1707006

87 Li M, Wei Q, Muduli SK, et al. Enhanced exciton and photon confinement in Ruddlesden-Popper perovskite microplatelets for highly stable low-threshold polarized lasing. Adv Mater, 2018, 30: 1707235

88 Lin $\mathrm{CH}$, Zeng Q, Lafalce E, et al. Large-area lasing and multicolor perovskite quantum dot patterns. Adv Opt Mater, 2018, 6: 1800474

89 Mathies F, Brenner P, Hernandez-Sosa G, et al. Inkjet-printed perovskite distributed feedback lasers. Opt Express, 2018, 26: A144

90 Nagamine G, Rocha JO, Bonato LG, et al. Two-photon absorption and two-photon-induced gain in perovskite quantum dots. J Phys Chem Lett, 2018, 9: 3478-3484

91 Pushkarev AP, Korolev VI, Markina DI, et al. A few-minute synthesis of $\mathrm{CsPbBr}_{3}$ nanolasers with a high quality factor by spraying at ambient conditions. ACS Appl Mater Interfaces, 2019, 11: $1040-1048$

92 Zhang S, Shang Q, Du W, et al. Strong exciton-photon coupling in hybrid inorganic-organic perovskite micro/nanowires. Adv Opt Mater, 2018, 6: 1701032

93 Zhao W, Qin Z, Zhang C, et al. Optical gain from biexcitons in $\mathrm{CsPbBr}_{3}$ nanocrystals revealed by two-dimensional electronic spectroscopy. J Phys Chem Lett, 2019, 10: 1251-1258

94 Huang C, Sun W, Liu S, et al. Highly controllable lasing actions in lead halide perovskite- $\mathrm{Si}_{3} \mathrm{~N}_{4}$ hybrid micro-resonators. Laser Photonics Rev, 2019, 13: 1800189

95 Liu Y, Yang W, Xiao S, et al. Surface-emitting perovskite random lasers for speckle-free imaging. ACS Nano, 2019, 13: 10653-10661
Wang K, Wang S, Xiao S, et al. Single-crystalline perovskite microlasers for high-contrast and sub-diffraction imaging. Adv Funct Mater, 2019, 29: 1904868

97 Zhang N, Fan Y, Wang K, et al. All-optical control of lead halide perovskite microlasers. Nat Commun, 2019, 10: 1770

98 Jia Y, Kerner RA, Grede AJ, et al. Diode-pumped organo-lead halide perovskite lasing in a metal-clad distributed feedback resonator. Nano Lett, 2016, 16: 4624-4629

99 Imamog $^{-}$lu A, Ram RJ, Pau S, et al. Nonequilibrium condensates and lasers without inversion: Exciton-polariton lasers. Phys Rev A, 1996, 53: 4250-4253

100 Deng H, Weihs G, Snoke D, et al. Polariton lasing vs. photon lasing in a semiconductor microcavity. Proc Natl Acad Sci USA, 2003, 100: 15318-15323

101 Evans TJS, Schlaus A, Fu Y, et al. Continuous-wave lasing in cesium lead bromide perovskite nanowires. Adv Opt Mater, 2018, 6: 1700982

102 Jiang L, Liu R, Su R, et al. Continuous wave pumped single-mode nanolasers in inorganic perovskites with robust stability and high quantum yield. Nanoscale, 2018, 10: 13565-13571

103 Jia Y, Kerner RA, Grede AJ, et al. Continuous-wave lasing in an organic-inorganic lead halide perovskite semiconductor. Nat Photon, 2017, 11: 784-788

104 Li Z, Moon J, Gharajeh A, et al. Room-temperature continuouswave operation of organometal halide perovskite lasers. ACS Nano, 2018, 12: 10968-10976

105 Brenner P, Bar-On O, Jakoby M, et al. Continuous wave amplified spontaneous emission in phase-stable lead halide perovskites. Nat Commun, 2019, 10: 988

106 Wang L, Meng L, Chen L, et al. Ultralow-threshold and colortunable continuous-wave lasing at room-temperature from in situ fabricated perovskite quantum dots. J Phys Chem Lett, 2019, 10: 3248-3253

107 Brenner P, Paetzold UW, Turnbull GA, et al. Comment on "Room-temperature continuous-wave operation of organometal halide perovskite lasers". ACS Nano, 2019, 13: 12257-12258

108 Sutherland BR, Hoogland S, Adachi MM, et al. Perovskite thin films via atomic layer deposition. Adv Mater, 2015, 27: 53-58

109 Miao Y, Ke Y, Wang N, et al. Stable and bright formamidiniumbased perovskite light-emitting diodes with high energy conversion efficiency. Nat Commun, 2019, 10: 3624

110 Huang X, Guo Q, Yang D, et al. Reversible 3D laser printing of perovskite quantum dots inside a transparent medium. Nat Photonics, 2020, 14: 82-88

Acknowledgements This work was financially supported by the $\mathrm{Na}$ tional Natural Science Foundation of China (61722502 and 61705009) and BIT funds.

Author contributions Wang L, Zhong $\mathrm{H}$ conceived the study and organized the manuscript; Dai G, Deng L contributed to discussion and revised the manuscript.

Conflict of interest The authors declare that they have no conflict of interest. 


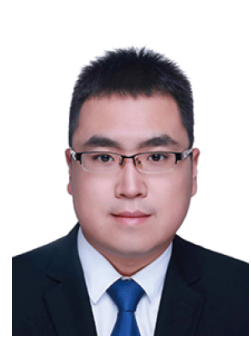

Lei Wang received his $\mathrm{PhD}$ degree of physics in 2017 from Beijing Institute of Technology (BIT). Currently, he is working as a postdoc with Prof. Haizheng Zhong in the School of Materials Science \& Engineering at BIT. His projects focus on QD-based laser for photonic and sensor applications.

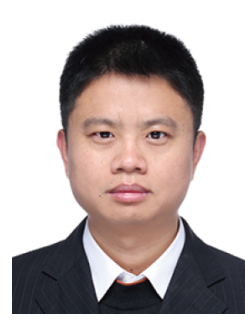

Haizheng Zhong is a full professor of the School of Materials Science \& Engineering at BIT. He joined BIT as an associate professor in 2010 and was promoted to full professor in 2013. His current research interests are in the area of CQDs for photonics and optoelectronics. Since 2019, he has served as a senior editor for The Journal of Physical Chemistry Letters. His recent awards include Beijing Science and Technology (2nd, 2/ 10), IDW 2019 best paper award.

\section{半导体量子点连续原浦激光研究进展}

王雷 ${ }^{1^{*}}$, 戴光 ${ }^{2}$, 邓罗根 ${ }^{2}$, 钟海政 ${ }^{{ }^{*}}$

摘要 连续激光器是光子芯片的关键部件. 低阈值、高效率、长寿 命、低成本、易集成的连续激光器一直是科学界和工业界追求的 目标之一. 量子点具有苂光量子产率高、发光波长可调、易于集 成等优点, 是一种优异的激光增益材料. 根据制备工艺的不同, 量 子点可分为外延生长的量子点和溶液制备的胶体量子点. 本文综 述了使用外延生长方法制备的III-V半导体量子点和胶体量子点连 续激光器的研究进展, 详细讨论了胶体量子点实现连续激光所面 临的困难和挑战, 总结了钻钛矿连续波激光器研究的进展. 最后, 讨论了量子点连续激光器实现光子芯片集成应用所面临的挑战. 